\title{
Psoriatic spondyloarthropathy: a long term prospective study
}

\author{
JOHN G HANLY, M LYNN RUSSELL, AND DAFNA D GLADMAN \\ From the University of Toronto Rheumatic Disease Unit, Women's College Hospital, Toronto, Canada
}

SUMMARY Fifty two patients with psoriatic spondyloarthropathy were monitored prospectivel over a mean of 57 months (range 30-107). A comprehensive protocol was used to assess clinica? and radiological features of disease activity and severity. Serial radiographs showed a significantN increase in the number of patients with syndesmophyte formation and sacroiliitis. In contrast $t_{\omega}^{\mathrm{N}}$ there was no significant increase in the number of patients with inflammatory neck pain oळ

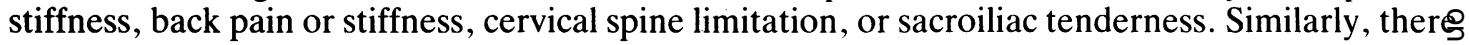
were no significant changes in any of the direct or indirect measurements of thoracolumbar spinemobility. The presence of HLA-B27 did not appear to influence disease progression. Thes results suggest that although patients with psoriatic spondyloarthropathy have radiologica progression of their disease, this remains clinically silent and does not compromise spina $\vec{\delta}_{\circ}^{\circ}$ mobility.

Key words: psoriatic arthritis, spinal disease, HLA-B27.

Clinical and radiological features of spinal disease have been reported in $20-40 \%$ of patients with psoriatic arthritis. ${ }^{1-12}$ The severity of symptoms secondary to spinal disease and the associated restriction in spinal mobility, however, are variable. Some cross sectional studies have reported a clinical pattern which is similar to that seen in ankylosing spondylitis, ${ }^{24-6}$ whereas others have emphasised the relative absence of symptoms and their lack of correlation with radiological changes. ${ }^{78}$ The latter include many unusual, although not unique features, such as asymmetric sacroiliitis and paramarginal syndesmophytes. ${ }^{17-9}$ The prevalence of the HLA-B27 antigen is increased in patients with psoriatic spondyloarthropathy, particularly those with sacroiliitis. ${ }^{1-3} 10$

The long term outcome of patients with psoriatic spondyloarthropathy has not been well documented. In particular, little is known of the changes in symptoms of spinal disease or in spinal mobility with time, and their correlation with changes in radiological features. Furthermore, the influence of other variables, such as peripheral joint disease and

Accepted for publication 8 October 1987.

Correspondence to Dr Dafna D Gladman, Women's College Hospital, Burton Hall, Suite 423, 60 Grosvenor St, Toronto, Ontario M5S 1B6, Canada.

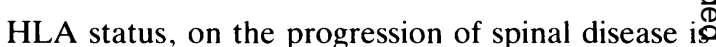
unknown.

The aim of the present study was to examine clinical and radiological features of spinal disease prospectively in a large group of patient enrolled in. a long term evaluation of psoriatic arthritis. Particu@ lar attention was paid to changes in spinal mobilito and their correlation with radiological progressions and HLA status.

\section{Patients and methods}

In 1978 a psoriatic arthritis clinic was established $a$ Women's College Hospital, Toronto. All patients enrolled in the clinic fulfilled the currently accepteos criteria for psoriatic arthritis,- - namely, the pre sence of an inflammatory arthropathy, usuallर rheumatoid factor negative, associated with psoriasis. ${ }^{11}$ Patients are evaluated on a regular basis using a comprehensive data retrieval protocol, the details of which have been published elsewhere. $\stackrel{100}{9}$ This protocol is used to monitor accurately clinical + radiological, and laboratory features of overals disease activity and severity. Particular care is takerep to exclude other possible causes of articular symp $\frac{?}{\mathbb{P}}$ toms, such as fibrositis and degenerative disc dis ease. The present study includes those patients wh\& showed clinical or radiological evidence of spina 
disease at their initial assessment, and in whom there was a minimum follow up of 30 months. The duration of follow up was arbitrarily selected in an attempt to obtain a clinically meaningful interval between assessments. Although most patients had several evaluations over the period of study, the data presented here are confined to the initial and final assessments.

Patients were included if any one of the following criteria were fulfilled: inflammatory neck pain or stiffness, inflammatory back pain or stiffness, or clinical sacroiliitis. The last was recorded by either the Gaenslen test (eliciting pain in the sacroiliac area when the patient's leg is allowed to drop over the edge of the examining table while the other leg is flexed), the Patrick-fabere test (eliciting pain in the sacroiliac area when pressure is applied to the pelvis on one side and to the flexed knee on the other, while the hip is placed in extreme flexion, abduction, and external rotation), or by direct pelvic compression. ${ }^{13}$ Radiological criteria included the presence of sacroiliitis, as defined by stages 1-4 of the New York criteria, ${ }^{14}$ or marginal or paramarginal syndesmophytes, or both. ${ }^{\top}$ Marginal syndesmophytes were recorded if the vertebral ossifications arose from the edge of the vertebral body and formed a fine vertical bridge, ${ }^{715}$ and paramarginal syndesmophytes were described when the ossification arose away from the edge of the vertebra and was broad and coarse. ${ }^{9}$ Syndesmophytes were distinguished from osteophytes in that the latter, which originate from the cartilaginous end plate in response to degeneration of the disc, are wider, horizontally oriented, and are associated with narrowed disc spaces. ${ }^{15}$ Paramarginal syndesmophytes were also distinguished from diffuse idiopathic skeletal hyperostosis (DISH) in that the latter condition occurs in four or more consecutive vertebrae, involves primarily the right side of the thoracic spine, and is associated with normal sacroiliac joints. ${ }^{16}$ All radiographs were read by at least two rheumatologists. There was complete agreement between the readers in the films of 49 of the 52 patients, and a consensus was achieved for the remaining three films. One of the rheumatologists read all the films on two separate occasions, without knowing the identity of the patients. The grading of the sacroiliac joints was identical in all but one film taken at initial assessment, which was originally read as grade 2 and on the second reading was graded 3. Thus reproducibility was shown in $51 / 52$ of the cases.

The following indices of spinal disease were recorded at each assessment: presence of inflammatory pain or stiffness involving the cervical or thoracolumbar spine, qualitative restriction in cer- vical mobility, ${ }^{17}$ measurement of thoracolumbar mobility, clinical sacroiliitis by the three manoeuvres already described, and radiographs of the cervical and thoracolumbar spine (anteroposterior and lateral views) and sacroiliac joints (anteroposterior view of the pelvis). Thoracolumbar mobility was measured by finger to floor distance, $10 \mathrm{~cm}$ segments test ${ }^{18}$ finger to fibula distance, and chest expansion.

Peripheral joint disease activity was measured by the duration of morning stiffness, grip strength, and American Rheumatism Association (ARA) joint count. ${ }^{19}$ The number of deformed joints (ankylosis, subluxation, or decreased range of motion attributable to joint damage rather than activity) was also recorded. Radiographs of the hands and feet were obtained and evaluated using the ARA staging criteria ${ }^{20}$ Laboratory evaluation included full HLA typing. ${ }^{21}$

Most patients (39/52) were taking non-steroidal anti-inflammatory drugs over the period of study. A few patients were also receiving one of a variety of 'disease suppressive' drugs, including intramuscular gold salts (three patients), oral methotrexate (four patients), chloroquine (one patient), and retinoic acid (four patients).

The data obtained were entered into a HewlettPackard 3000 computer. Statistical analysis was carried out using the SPSS (statistical package for social sciences) program. This included Student's $t$ test and one way analysis of variance for continuous variables, McNemar's $\chi^{2}$ and Fisher's exact test for comparing the frequency between different patient groups, and regression analysis for examining correlations between continuous variables.

\section{Results}

Fifty two patients were identified from a total clinic population of 220 patients (Table 1 ). There was a slight male predominance (male:female $=30: 22$ ). The mean age on entry to the study was 44 years and the mean duration of psoriatic arthritis was eight years. The duration of follow up varied from 30 to 107 months with a mean of 57 months.

Table 1 Clinical features of 52 patients with psoriatic spondyloarthropathy on entry to the study

\begin{tabular}{lr}
\hline Number of patients & 52 \\
Men:women & $30: 22$ \\
Mean age, years (range) & $44(23-70)$ \\
Mean disease* duration, years (range) & $8(0 \cdot 1-36)$ \\
Mean follow up, months (range) & $57(30-107)$
\end{tabular}

${ }^{*}$ Psoriatic arthritis. 
Table 2 Features of psoriatic spondyloarthropathy present at study entry in 52 patients

\begin{tabular}{lr}
\hline Features & Number of patients (\%) \\
\hline Axial symptoms & $34(65)$ \\
Neck pain or stiffness & $22(42)$ \\
Back pain or stiffness & $25(48)$ \\
Neck and back pain or stiffness & $13(25)$ \\
Sacroiliitis (clinical) & $9(17)$ \\
Syndesmophytes & $14(27)$ \\
Cervical & $20(38)$ \\
Thoracolumbar & $13(25)$ \\
Sacroiliitis (radiological) & \\
stage 0 & $15(29)$ \\
1 & $9(17)$ \\
2 & $11(21)$ \\
3 & $4(8)$ \\
4 &
\end{tabular}

${ }^{*}$ The highest score of the two sacroiliac joints was taken for each patient.

Table 2 shows the number of patients fulfilling the different selection criteria. Thirty four patients had symptoms of inflammatory spinal disease. Inflammatory neck pain or stiffness and inflammatory back pain or stiffness were present in 22 and 25 patients respectively, while 13 patients had both neck and back symptoms. Clinical evidence of sacroiliitis was present in nine patients. Twenty two patients had syndesmophytes at one or more spinal levels, which were frequently in a 'spotty' asymmetric distribution. Twenty patients had syndesmophytes in the thoracolumbar spine, 14 had disease in the cervical spine, two of whom had isolated cervical spine syndesmophytes. Twelve patients had marginal syndesmophytes only, five patients had paramarginal syndesmophytes only, and the remaining five patients had a combination of both. Definite radiological sacroiliitis, as defined by stage 2 or more of the New York criteria, ${ }^{14}$ was present in 24 of the patients, with equivocal changes in 15. Only four patients had total ankylosis of one or both sacroiliac joints. A total of $8 / 24$ patients with definite sacroiliitis had symmetrical disease. The correlation between radiological sacroiliitis and syndesmophyte formation was also examined. Thirteen of the 24 patients with definite sacroiliitis had syndesmophyte formation at one or more spinal levels. Conversely, of the 22 patients with syndesmophytes, 13 had definite radiological sacroiliitis (grades 2-4), seven had grade 1 sacroiliitis, and two were considered to have normal sacroiliac joints. Both patients had paramarginal syndesmophytes only, as well as inflammatory back pain. Their syndesmophytes were single, and thus did not qualify as diffuse idiopathic skeletal hyperostosis. ${ }^{16}$
Table 3 Changes in symptoms of spinal disease over 30-107 months in 52 patients with psoriatic spondyloarthropathy

\begin{tabular}{lcc}
\hline Symptom & Entry $^{*}$ & Follow up* $^{*}$ \\
\hline Neck pain & 17 & 21 \\
Neck stiffness & 19 & 20 \\
Back pain & 20 & 14 \\
Back stiffness & 23 & 19 \\
Sacroiliitis (clinical) & 9 & 3
\end{tabular}

*Number of patients is shown.

Table 3 shows the changes in symptoms of spinal disease over the period of study. There was little change in the number of patients with cervical spine symptoms. The number of patients with inflamma- $\stackrel{\infty}{\circ}$ tory back pain and stiffness and clinical sacroiliitis 0 fell, but this did not reach statistical significance.

Table 4 summarises the changes in spinal mobility $z$ over the same period. The number of patients with restricted cervical spine mobility did not change $\vec{\oplus}$ significantly. Similarly, thoracolumbar mobility was $\infty$ unaltered as reflected by almost identical measurements for finger to floor distance, $10 \mathrm{~cm}$ segments test, finger to fibula distance, and chest expansion at both assessments.

In contrast with the preservation of spinal mobil-。ू๊ ity, serial radiographs showed radiographic progres- $\frac{\mathbb{}}{2}$ sion of spinal disease; Table 5 summarises these $\overrightarrow{\overrightarrow{0}}$ findings. Follow up films were unavailable for six patients, which accounts for the numerical discrepancies between Tables 2 and 5. Syndes-? mophytes, predominantly in the thoracolumbar spine, appeared for the first time over the study? period in six patients. An additional three patients, who had syndesmophytes at multiple levels at their initial assessment, also showed progression of syndesmophyte formation over the study period. This progression included the formation of syndes-

Table 4 Changes in spinal mobility over 30-107 months in 52 patients with psoriatic spondyloarthropathy

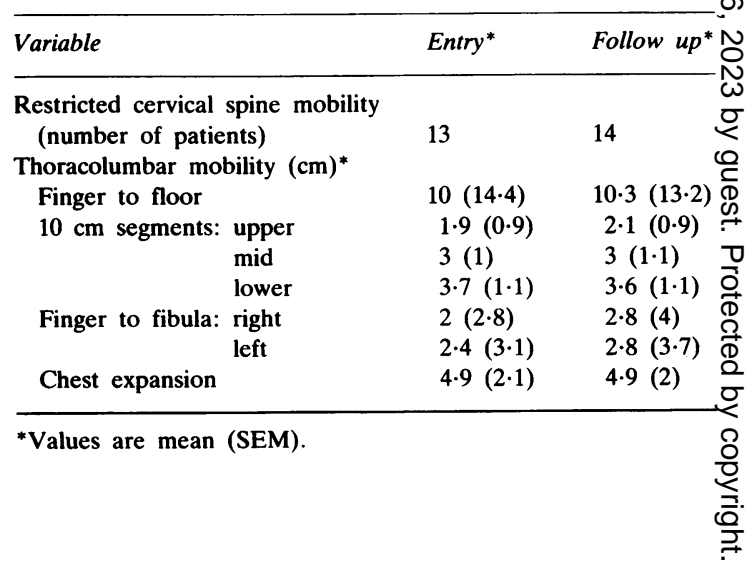


mophytes at new spinal levels and enlargement of pre-existing syndesmophytes. Radiological progres-

Table 5 Changes in radiological features of psoriatic spondyloarthropathy over 30-107 months in 52 patients

\begin{tabular}{|c|c|c|c|c|}
\hline \multirow{2}{*}{$\begin{array}{l}\text { Radiological } \\
\text { feature } \\
\text { Syndesmophytes }\end{array}$} & \multicolumn{2}{|c|}{ Entryt } & \multicolumn{2}{|c|}{ Follow up $\dagger$} \\
\hline & \multirow{3}{*}{\multicolumn{2}{|c|}{$\begin{array}{l}13 \\
18\end{array}$}} & \\
\hline Cervical & & & 14 & \\
\hline \multirow[t]{3}{*}{ Thoracolumbar } & & & $24^{*}$ & \\
\hline & \multicolumn{2}{|c|}{$R i g h t^{* *}$} & \multicolumn{2}{|c|}{$L e f t^{* * *}$} \\
\hline & Entry & Follow up & Entry & Follow up \\
\hline \multicolumn{5}{|l|}{ Sacroiliitis } \\
\hline Stage 0 & 15 & 12 & 15 & 13 \\
\hline 1 & 12 & 7 & 12 & 7 \\
\hline 2 & 7 & 8 & 7 & 9 \\
\hline 3 & 8 & 14 & 9 & 13 \\
\hline 4 & 4 & 5 & 3 & 4 \\
\hline
\end{tabular}

${ }^{*} \mathrm{p}=0.03$ (McNemar's $\chi^{2}$ test); ${ }^{* *} \mathrm{p}=0.0002$ (reflecting changes in right sacroiliac joint); ${ }^{* * *} \mathrm{p}=0.002$ (reflecting changes in left sacroiliac joint).

†Number of patients is shown.

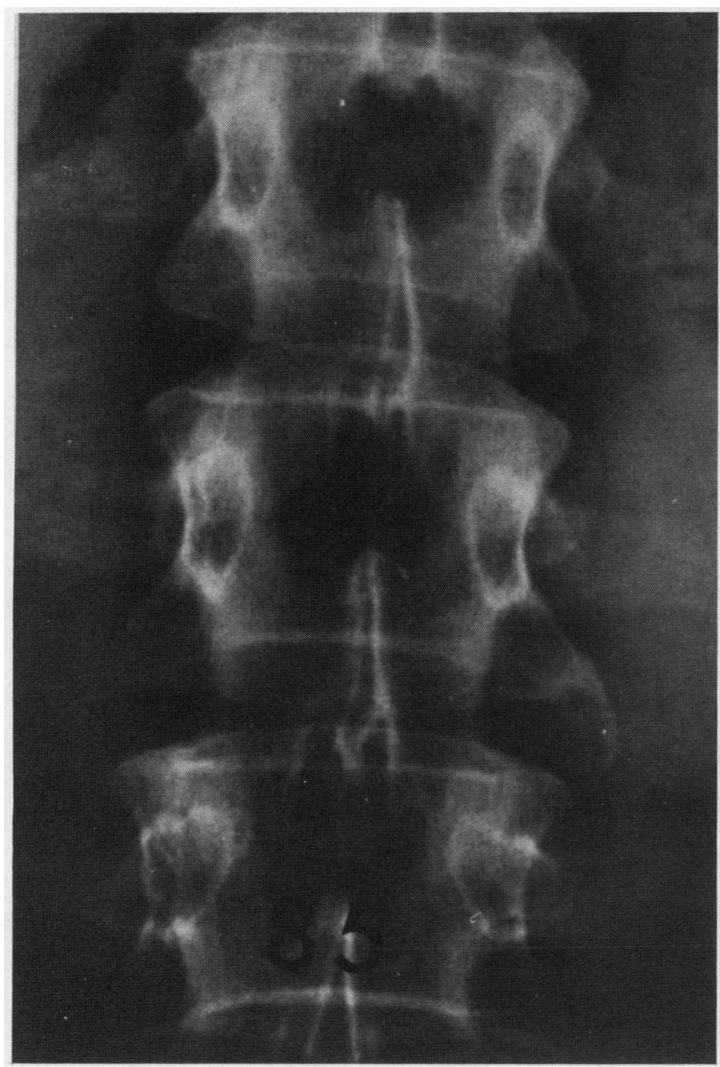

Fig. 1a sion was equally common for marginal and paramarginal syndesmophytes. Sacroiliitis also progressed significantly over the study period. Table 5 shows changes in the grades of sacroiliitis for each side. Although significance of the change on the right side was stronger than that on the left $(\mathrm{p}=0.0002 v \mathrm{p}=0.002)$, there was equal representation of both sacroiliac joints in these changes. Most changes occurred within stages 1 to $3 .^{14}$ Thus although there was a reduction of the number with grade 1 sacroiliitis at follow up compared with entry, grade 3 changes were more prevalent at the last assessment. Of the 16 patients who showed progression in at least one sacroiliac joint, 11 progressed by one stage, two progressed by two stages, and three progressed by three stages. Figs 1-3 show selected examples of radiological progression. As skeletal ossification has been reported as a complication of retinoic acid derivatives ${ }^{22}$ it is important to note that none of the patients who had taken retinoic acid showed progression of spinal disease.

Although there was no change in the clinical

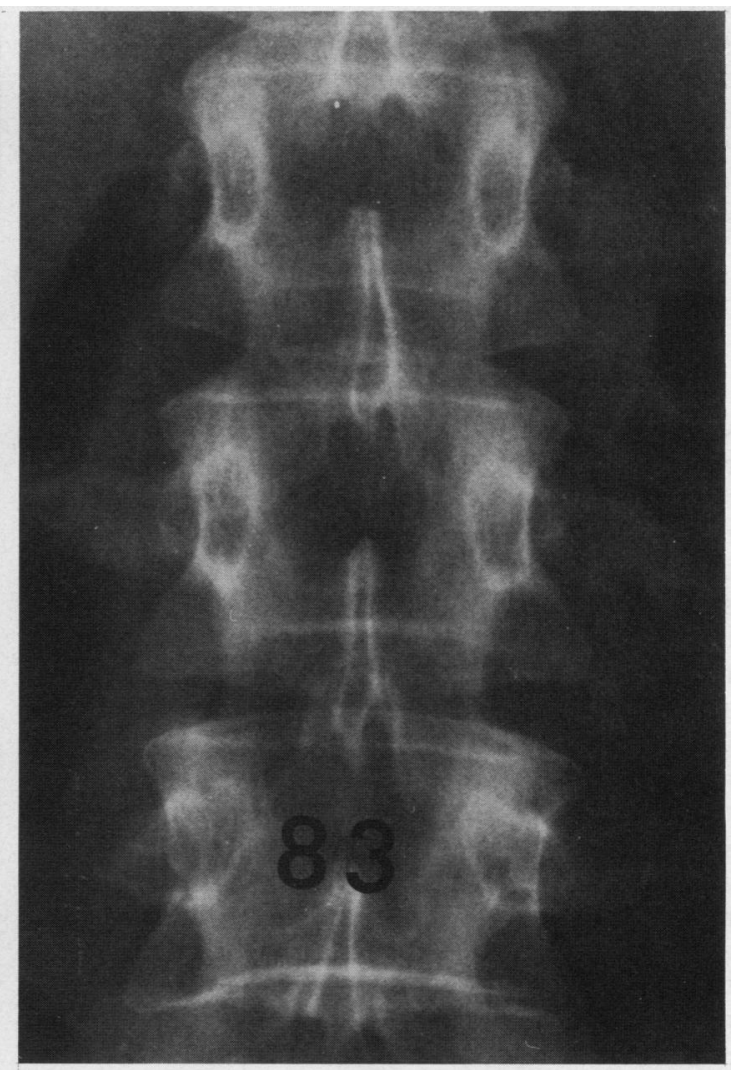

Fig. 1b

Fig. 1 (a) and (b) Enlargement over three years of an isolated paramarginal syndesmophyte in the lumbar spine. 


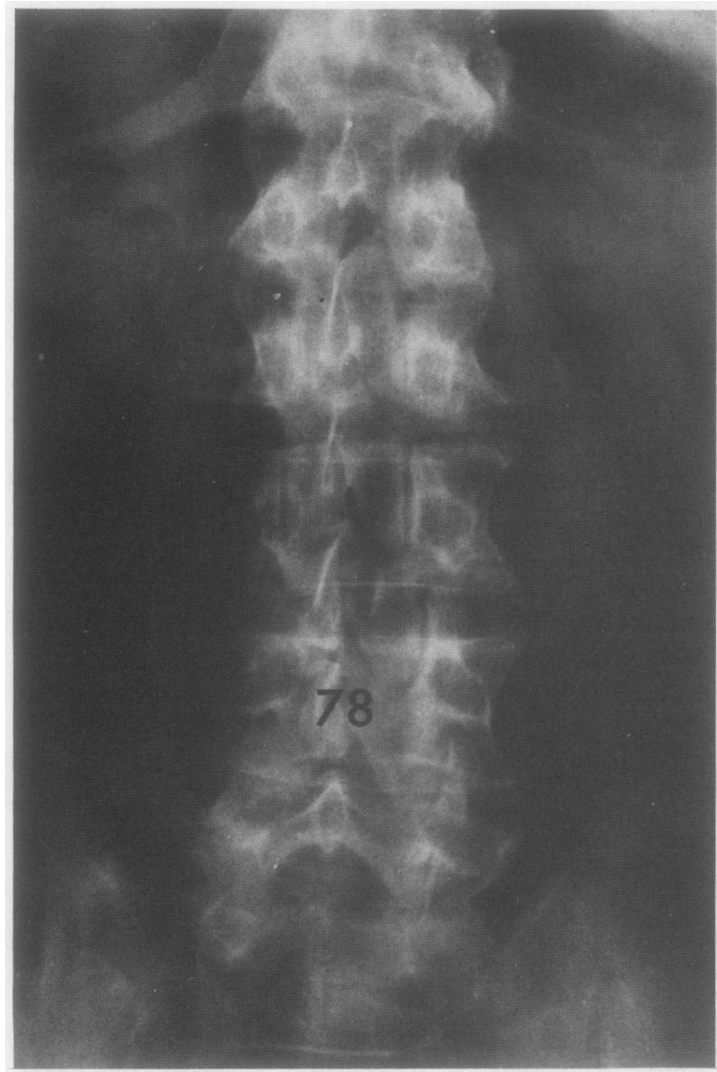

Fig. 2a

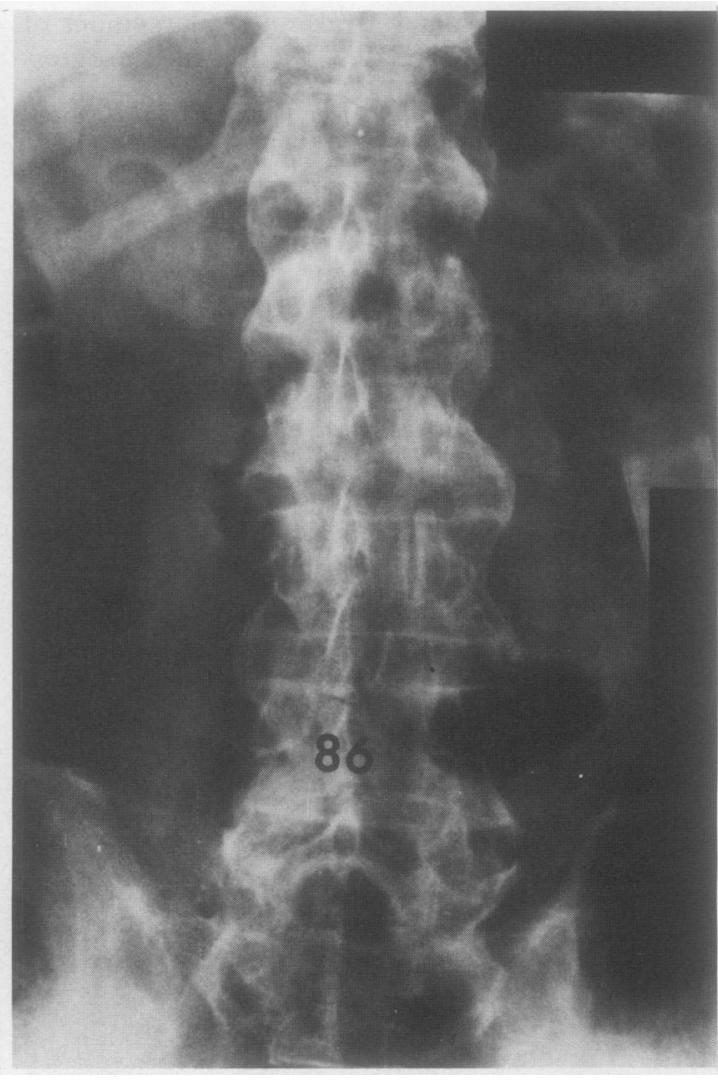

Fig. $2 b$

Fig. 2 (a) and (b) Enlargement over eight years of marginal and paramarginal syndesmophytes in the lumbar spine.

variables of spinal disease in the entire study group, there was a possibility that changes might have occurred in various subgroups of patients, such as those with radiological progression or the longest duration of follow up. Therefore, to ensure that such changes were not being overlooked, in particular with regard to spinal mobility, the data were reanalysed. Firstly, those patients who developed symptoms of spinal disease or who showed radiological progression over the study period were identified. With the exception of one patient who developed 'a classic 'bamboo spine', there were no significant changes in any index of spinal mobility in either group. Similarly, when the effects of other potentially confounding variables, such as patient age, age at onset of arthritis, disease duration, and the duration of follow up, were examined there was no divergence from the data already presented.

It was of interest to examine how concurrent peripheral joint disease and HLA status may correlate with or influence changes in clinical and radiological variables of spinal disease. There was a significant increase in peripheral joint damage over the study period as reflected by an increase in the $\frac{3}{3}$ clinically deformed joint count (mean (SEM): 4.4 $(1.04)$ to $6.5(1.44), p=0.006, t$ test) and in the number of joints with radiological erosions (mean $\frac{T}{0}$ (SEM): $1 \cdot 30(0 \cdot 37)$ to $2 \cdot 5(0 \cdot 44), \mathrm{p}=0 \cdot 004, t$ test $)$ and ankylosis (mean (SEM): $2 \cdot 7(0 \cdot 88)$ to $3.6(1 \cdot 11)$, స $\mathrm{p}=0.036, t$ test $)$. These changes, however, did not $N$ correlate with changes in either clinical or radio- N logical variables of spinal disease over the same $\omega$ period. Similarly, there was no correlation between any pattern of peripheral joint disease (e.g., distal $\stackrel{0}{=}$ interphalangeal joint only, oligoarthritis, poly- $\stackrel{\oplus}{\oplus}$ arthritis $^{12}$ ) and progression of spinal disease. HLAB27 was present in 11/47 of the patients. The $\overrightarrow{0}$ strongest association was with the presence of $\overrightarrow{\mathbb{D}}$ syndesmophytes, where $7 / 22$ of the patients were $\frac{?}{\mathbb{D}}$ HLA-B27 positive, rather than with radiological $\cong$ sacroiliitis ( $\geqslant$ stage 2 of the New York criteria ${ }^{14}$ ), where $7 / 29$ of the patients were HLA-B27 positive. 8 


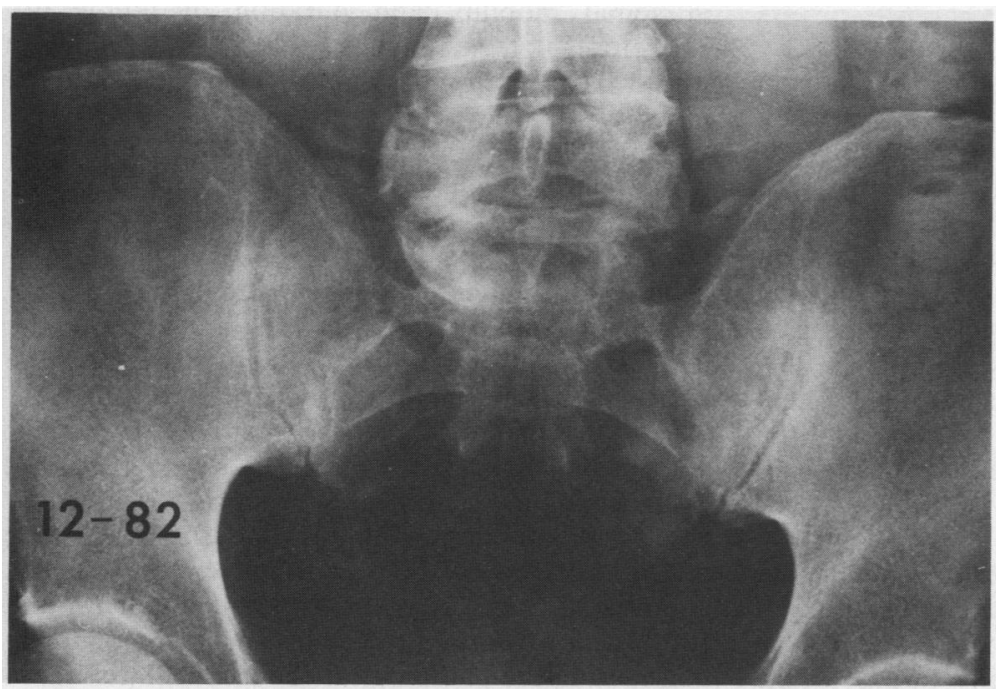

Fig. 3a

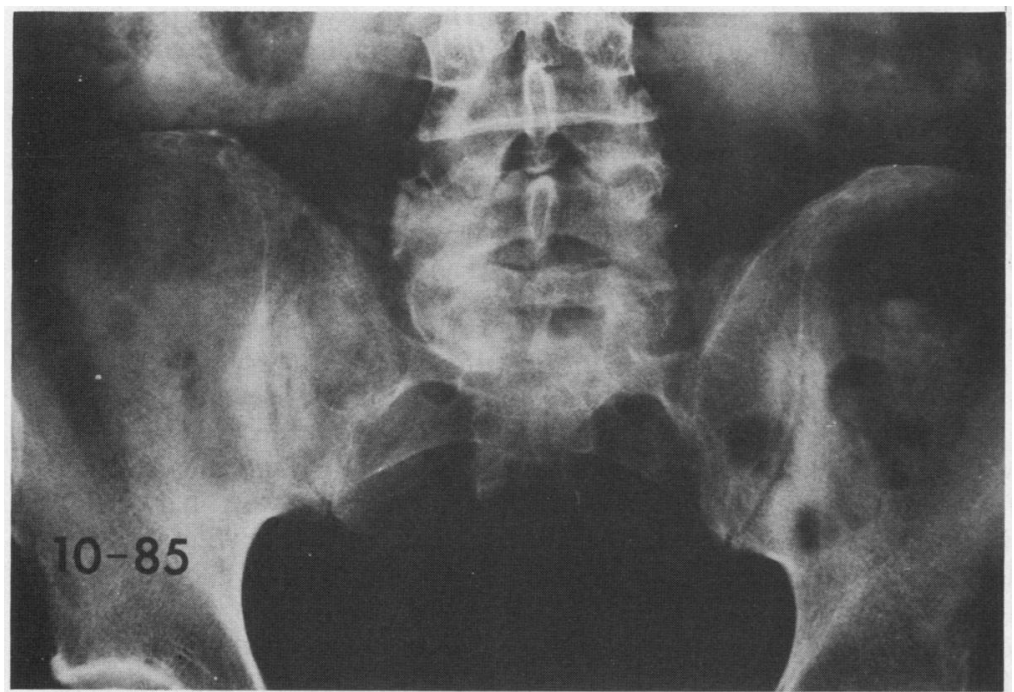

Fig. 3b

There was no correlation between any HLA antigen, including HLA-B27, and changes in clinical variables of spinal disease. Table 6 summarises the influence of HLA-B27 on the radiological progression of spinal disease. There was no difference in the progression of sacroiliitis between the HLA-B27 positive and negative groups. Progression in cervical spine syndesmophyte formation was more common in HLA-B27 negative patients, whereas thoracolumbar syndesmophyte formation occurred more frequently in HLA-B27 positive patients. The number of patients involved was small, however, and these differences did not reach statistical significance.
Fig. 3 (a) and (b) Progression over 34 months of bilateral sacroiliitis from stage 1 to stage 3 of the New York criteria. ${ }^{14}$
Table 6 Influence of HLA-B27 on radiological progression of psoriatic spondyloarthropathy over 30-107 months

\begin{tabular}{|c|c|c|}
\hline \multirow[t]{2}{*}{ Radiological progression } & $H L A-B 27+$ & $H L A-B 27-$ \\
\hline & $n(\%)$ & $n(\%)$ \\
\hline \multicolumn{3}{|l|}{ Syndesmophytes } \\
\hline Cervical & $0 / 11^{*}$ & $3 / 33(9)$ \\
\hline Thoracolumbar & $3 / 11(27)$ & $6 / 36$ (17) \\
\hline Sacroiliitis & $4 / 11(36)$ & $12 / 35(34)$ \\
\hline
\end{tabular}

*Values show number with progression/number with that particular tissue type. 


\section{Discussion}

Although the concept of spinal involvement in patients with psoriatic arthritis is well established, ${ }^{1-10}$ there is little information concerning its long term outcome. This study examines the changes in clinical and radiological features of spinal disease in 52 patients with psoriatic spondyloarthropathy over a mean of 57 months (range 30-107). Patients were monitored prospectively using a comprehensive protocol in the setting of a fully dedicated research clinic. The results suggest that despite radiological progression there was little change in symptoms of spinal disease and, perhaps most important of all, that spinal mobility was well preserved.

The design of the psoriatic arthritis clinic at our hospital has been described in detail elsewhere. ${ }^{12} 21$ The clinic population reflects the spectrum of manifestations of psoriatic arthritis owing to the wide based source of referrals, the rigorous exclusion of other causes of arthropathy, and the use of an identical comprehensive protocol to evaluate all patients. In a previous report of the first 220 patients enrolled in the clinic the prevalence of psoriatic spondyloarthropathy was $33 \% .^{12}$ The present study of 52 (a prevalence of $24 \%$ ) patients excludes those whose follow up was less than 30 months' duration. Although the presence of spinal disease in psoriatic arthritis has been reported with variable frequency, most large studies have reported a prevalence in the range of $20-40 \%,{ }^{1-4}$ in keeping with our own findings. The slight male predominance of our patients has also been noted by others. ${ }^{1}$

An important finding in our study was the lack of progression in clinical features of spinal disease as reflected by either symptoms of spondyloarthropathy or changes in spinal mobility. There are a number of possible explanations to account for this observation. Firstly, the duration of follow up might have been insufficient to allow full expression of spinal disease. Although this can only be determined with certainty after further follow up, it is of interest to note the findings of a study of 51 patients with ankylosing spondylitis who were followed up over a mean of 38 years. ${ }^{23}$ In this group the pattern of restriction in spinal mobility became apparent within the first 10 years of follow up and in most patients it did not alter significantly after this time. In an unrelated study of psoriatic spondyloarthropathy there was little change in either the number of patients with syndesmophytes or the number of syndesmophytes per patient when disease duration exceeded 10 to 15 years. ${ }^{7}$ In the present study the maximum duration of follow up was almost nine years and there was no correlation between changes in spinal mobility and duration of follow up믄 Therefore, it seems unlikely that further follow up? would have disclosed significant changes in spina $\overrightarrow{5}$ mobility in the majority of our patients.

An alternative explanation is that spinal mobilityo was already maximally restricted by the time of the initial assessment and that a prolonged observation period would, therefore, not show any further changes. Although there are no well defined norma? values adjusted for age, sex, and body size for the $\vec{\circ}$ indices of spinal mobility used in this study, the mean values in Table 4 would probably be con sidered normal by most rheumatologists familiae with these techniques. ${ }^{18}$ Furthermore, the progres? sion in radiological features of spinal disease over the period of study suggests that the underlyingr pathological process was still active.

Perhaps the most plausible explanation for theo preservation of spinal mobility may be found by considering the pathological changes themselves, in particular the characteristics and distribution of the syndesmophytes. The 'spotty' asymmetric distribu= tion of marginal and paramarginal syndesmophyte 300 in our patients is similar to that in previous descriptions of psoriatic spondyloarthopathy ${ }^{17}$ and is in contrast with the typical findings of ankylosing spondylitis. ${ }^{7}$ Other important distinguishing features are the relative absence of ligamentous ossifio cation and sparing of the apophyseal joints by the® inflammatory process. ${ }^{7}$ Although marginal syndes $\overrightarrow{\vec{*}}$ mophytes reflect ossification of the superficial layer of the fibrous ring of the intervertebral disc, ${ }^{24}$ paramarginal syndesmophytes are due to ossifica? tion in tissues further out from the annulus. ${ }^{79}$ Thein exact pathogenesis is unknown, but they may resule $\bar{\rho}$ from an inflammatory process in paravertebra? connective tissue, ${ }^{1}$ or from a resolving periostitis. Whatever their aetiology, it is probable that their intrinsic properties and distribution, in conjunction with the relative lack of involvement of other spinaB structures, may result in fewer symptoms of spinat disease and a normal range of spinal mobility.

There is a strong association between inflamma tory back disease and the presence of HLA-B27 particularly in ankylosing spondylitis. ${ }^{25} 26$ The asso $\mathrm{N}$ ciation with psoriatic spondyloarthropathy is lessw impressive. The reported prevalence ranges frome $21 \%$ to $76 \%,{ }^{110}$ and the strongest association is with sacroiliitis. ${ }^{110}$ In the present study HLA-B2 was detected in $11 / 47(23 \%)$ of the total study group ${ }^{+}$ and showed the strongest association with the presence of syndesmophytes. These findings may be्ष partly affected by the relatively small sample size, as? in a previous study of 158 patients in our clinio HLA-B27 was detected in $37 \%$ of patients with spinal disease. ${ }^{21}$ The presence of HLA-B27 did noto 
appear to influence either clinical or radiological progression of disease. Similarly, although peripheral joint disease progressed over the study period, it did not correlate with changes in spinal disease. This suggests that spinal disease and peripheral joint disease in patients with psoriatic arthritis behave independently of each other.

The authors wish to thank Professor Rob Roberts of the Department of Clinical Epidemiology and Biostatistics, McMaster University, Hamilton, Ontario, for his assistance in statistical analysis. This work was supported in part by the Canadian Arthritis Society.

\section{References}

1 Lambert J R, Wright V. Psoriatic spondylitis: A clinical and radiologic description of the spine in psoriatic arthritis. $Q J \mathrm{Med}$ 1977; 46: 411-25.

2 Kammer G M, Soter N K, Gibson D J, Schur P H. Psoriatic arthritis: a clinical, immunologic and HLA study of 100 patients. Semin Arthritis Rheum 1979; 9: 75-97.

3 Leonard D G, O'Duffy J D, Rogers III R S: Prospective analysis of psoriatic arthritis in patients hospitalized for psoriasis. Mayo Clin Proc 1978; 53: 511-8.

4 Moll J M H, Wright V. Psoriatic arthritis. Semin Arthritis Rheum 1973; 3: 55-78.

5 Reed W B. Psoriatic arthritis. A complete clinical study of 86 patients. Acta Derm Venereol (Stockh) 1961; 41: 396-403.

6 Avila R, Pugh D G. Slocumb C H, Winkelman R K. Psoriatic arthritis: a roentgenological study. Radiology 1960; 75: 691-701.

7 McEwen C, Di Tata D, Lingg C, Porini A, Good A, Rankin T. Ankylosing spondylitis and spondylitis accompanying ulcerative colitis, regional enteritis, psoriasis and Reiter's discase. Arthritis Rheum 1971; 14: 291-318.

8 Jajic I. Radiological changes in the sacro-iliac joints and spinc of patients with psoriatic arthritis and psoriasis. Ann Rheum Dis 1968; 27: 1-6.

9 Bywaters E G L, Dixon A St J. Paravertebral ossification in psoriatic arthritis. Ann Rheum Dis 1965; 24: 313-31.

10 Barraclough D, Russell A S, Percy J S. Psoriatic spondylitis: a clinical, radiological and scintiscan study. $J$ Rheumatol 1977; 4: 282-7.
11 Wright V. Psoriatic arthritis. In: Kelly W N, Harris E D. Ruddy S, Sledge C B, eds. Textbook of rheumatology. 2nd ed, Philadelphia: Saunders, 1985: 1021-31.

12 Gladman D D, Shuckett R. Russell M L, Thorne J C. Schachter $\mathrm{R} \mathrm{K}$. Psoriatic arthritis-an analysis of 220 patients. $Q J$ Med 1987; 62: 127-41.

13 Hoppenfeld S. Physical examination of the spine and extremities. New York: Applcton-Century-Crofts, 1976: 261-2.

14 Bennett P H, Burch T A. New York symposium on population studies in the rheumatic diseases: new diagnostic critiera. Bull Rheum Dis 1967; 17: 453-8.

15 Riley M J, Ansell B M, Bywater E G L. Radiologic manifestations of ankylosing spondylitis according to age at onset. Ann Rheum Dis 1971; 30: 138-48.

16 Resnick D, Niwayama G. Radiographic and pathologic features of spinal involvement in diffuse idiopathic skeletal hyperostosis (DISH). Radiology 1976; 119: 559-68.

17 Hoppenfeld S. Physical examination of the spine and extremities. New York: Appleton-Century-Crofts, 1976: 114-6.

18 Miller M H, Lee P, Smythe H A. Goldsmith C H. Measurement of spinal mobility in the saggital plane: new skin contraction technique compared with established methods. J Rheumatol 1984; 11: 507-11.

19 Cooperating Clinics Committee of the American Rheumatism Association. A seven-day variability study of 499 patients with peripheral rheumatoid arthritis. Arthritis Rheum 1965; 8: 302-32.

20 Steinbrocker $\mathrm{O}$, Traeger $\mathrm{C} \mathrm{H}$, Batterman R C. Therapeutic criteria for rheumatoid arthritis. JAMA 1949; 140: 659-62.

21 Gladman D D, Anhorn K A B, Schachter R K. Mervart H. HLA antigens in psoriatic arthritis. $J$ Rheumatol 1986; 13: 586-92.

22 Pittsley R A, Yider F W. Retinoid hyperostosis. N Engl J Med 1983; 308: 1012-4.

23 Carette S, Graham D, Little H, Rubinstein J, Rosen P. The natural disease course of ankylosing spondylitis. Arthritis Rheum 1983; 26: 186-90.

24 Wetstein P, Riotton G. Aspects anatomoradiologiques de la spondylarthrite ankylosante. Radiol Clin North Am 1950; 19: 325-31.

25 Brewerton D A, Caffrey M. Hart F B. James D C O. Nicholls A, Sturrock R D. Ankylosing spondylitis and HL-A27. Lancet 1973; i: 904-7.

26 Schlosstein L, Terasaki P I, Bluestone R, Pearson C M. High association of an HLA antigen W27 with ankylosing spondylitis. $N$ Engl J Med 1973; 288: 704-6. 